\title{
Trait anxiety and dynamic adjustments in conflict processing
}

\author{
Roman Osinsky, Nina AleXander, Helge Gebhardt, ANd Juergen HenNig \\ Justus Liebig University, Giessen, Germany
}

\begin{abstract}
Recently, it has been assumed that high- and low-trait-anxious subjects differ in the way they use fundamental cognitive control mechanisms. The present study was designed to further elucidate this topic by focusing on trial-to-trial adjustments in neuronal correlates of conflict processing. An electroencephalogram was recorded while subjects $(N=71)$ performed a gender discrimination version of the Stroop task. The conflict-related N400 of the ERP was influenced by an interaction between trait anxiety and previous trial context: An additional negative-going deflection in the N400 range was observed when the target-distractor pairing of the directly preceding trial was incongruent, but only in highly anxious subjects. Thus, highly anxious subjects appear to more strongly engage neuronal modules involved in conflict monitoring when previously exposed to a high stimulus-response conflict. These results indicate that trait anxiety is crucially linked to the way the cognitive system dynamically adapts to recent demands.
\end{abstract}

Trait anxiety is probably not only specifically related to the processing of emotional material; it might also be generally linked to individual differences in fundamental cognitive functions. This has been suggested by studies demonstrating relations between trait anxiety and complex cognition, such as working memory (Derakshan \& Eysenck, 1998), set shifting (Ansari, Derakshan, \& Richards, 2008), and the capability to suppress distracting information (Eysenck \& Byrne, 1992; Wood, Mathews, \& Dalgleish, 2001).

In their influential attentional control theory (ACT), Eysenck, Derakshan, Santos, and Calvo (2007) therefore assumed that high trait anxiety disrupts the balance between goal-directed and stimulus-driven attentional systems (Corbetta \& Shulman, 2002), leading to an enhanced intrusion of task-irrelevant information. According to the ACT, behavioral consequences of such anxiety-related deficits in attentional guidance should emerge primarily in tasks requiring inhibitory control and mental set shifting (Miyake et al., 2000) by affecting reaction times (RTs; i.e., processing efficiency) but not accuracy (i.e., processing effectiveness). Moreover, anxious subjects may compensate for this efficiency deficit by a reactive recruitment of additional attentional resources if these are available.

A similar assumption has been put forward by Braver and colleagues (Braver, Gray, \& Burgess, 2007; Fales et al., 2008). In their dual mechanisms of control (DMC) account, these authors proposed a link between individual differences in trait anxiety and the general manner in which cognitive control is exerted. Accordingly, during a complex task, low-anxious subjects may engage top-down control in a proactive way by sustaining goal-directed rep- resentations in working memory over time. This should lead to a rather low influence of goal-irrelevant information. In contrast, highly anxious subjects are supposed to exert control in a reactive mode by reactivating goalrelevant representations only when these are needed. Consequently, bottom-up input will be more influential.

Although the ACT and DMC accounts apparently differ in some important aspects, they also share some essential ideas. For instance, both theories postulate a reactive and compensatory recruitment of cognitive resources in high-trait-anxious subjects when there is an acute need for top-down guidance. On the neuronal level, this should lead to a stronger transient activation of control networks, especially comprising prefrontal and parietal regions. The present study was planned to further elucidate the potential link between trait anxiety and the recruitment of cognitive control by focusing on dynamic adjustments in electrophysiological correlates of control mechanisms in a trial-to-trial design.

Using functional magnetic resonance imaging (fMRI), a few studies have already tested for trait-anxietyassociated differences in the activation of cognitive control areas. Unfortunately, they have not yet revealed a clear picture. For example, Braver and colleagues (Fales et al., 2008; J. R. Gray \& Braver, 2002) reported positive correlations between trait anxiety and transient activation in various control regions (particularly including the lateral and medial prefrontal cortex) during working memory performance. However, the same group reported no such relations in another study, despite use of the same working memory task (J. R. Gray et al., 2005). Moreover, Bishop (2009) found a negative relation between 
trait anxiety and transient activity of the dorsolateral prefrontal cortex (DLPFC) while subjects performed a task evoking stimulus-response conflicts.

It should be noted that the aforementioned studies completely disregarded trial-to-trial adaptations in cognitive control. For example, in paradigms evoking stimulusresponse conflicts (e.g., the Stroop task), it is a frequently observed phenomenon that interference is reduced in the context of a preceding incongruent trial (for an overview, see Egner, 2007). This observation is most likely due to adjustments in cognitive control: Upon detection of a stimulus-response conflict, control is enhanced and subsequently influences target-distractor processing within the following trial (Botvinick, Braver, Barch, Carter, \& Cohen, 2001; Carter \& van Veen, 2007; Egner \& Hirsch, 2005; Scerif, Worden, Davidson, Seiger, \& Casey, 2006). For instance, on incongruent trials, such sequencedependent enhancements in cognitive control probably lead to a diminished effect of the misleading distractor, resulting in performance improvements (i.e., faster responses and fewer errors on incongruent trials preceded by other incongruent, as compared with congruent, trials). However, on congruent trials, enhanced top-down control presumably diminishes the facilitative effect of the taskirrelevant dimension (which also indicates the correct response), leading to a decline in performance (i.e., slower responses and more errors on congruent trials preceded by incongruent, as compared with congruent, trials). Consideration of such dynamic adjustments may further our understanding of a potential link between trait anxiety and the reactive recruitment of top-down control. Due to its high temporal resolution, event-related potential (ERP) measurement appears to be particularly suitable for such an approach (Gratton, Coles, \& Donchin, 1992; Larson, Kaufman, \& Perlstein, 2009; Scerif et al., 2006).

In the present study, we therefore used ERP recordings to investigate the link between trait anxiety and sequential effects on conflict processing in a modified Stroop paradigm. In the classic version of this task (MacLeod, 1991; Stroop, 1935), color words are presented in varying colors, and the subject is asked to discriminate the color (target dimension) while ignoring the word meaning (distractor dimension). An incompatible target-distractor pairing (e.g., the word red written in blue) induces a stimulus-response conflict, resulting in a prolonged RT and a higher error rate. The neurocognitive mechanisms mediating the detection and resolution of such conflicts have been the subject of an extensive empirical effort. Many fMRI approaches addressing this issue point to a crucial role of the dorsal anterior cingulate cortex (dACC) in processes of conflict monitoring and of the DLPFC in the execution of control (Braver, Barch, Gray, Molfese, \& Snyder, 2001; Carter et al., 2000; Kerns et al., 2004; MacDonald, Cohen, Stenger, \& Carter, 2000; van Veen \& Carter, 2002). Importantly, activation in these areas also depends on sequential alternations of stimulus-response conflict, indicating dynamic adaptations to task demands (Egner \& Hirsch, 2005; Kerns et al., 2004). Moreover, several ERP studies have identified at least two characteristic wave components as electrophysiological correlates of conflict processing in the Stroop task. Accordingly, a high target-distractor conflict is usually accompanied by a frontocentral negative-going voltage deflection about $400 \mathrm{msec}$ after stimulus onset (N400), followed by a later, more posterior sustained positive potential (SP). The N400 has been assumed to reflect a conflict-monitoring and detection mechanism, and source-locating approaches have confirmed the above-mentioned fMRI results by demonstrating that this component is presumably generated by the dACC (Badzakova-Trajkov, Barnett, Waldie, \& Kirk, 2009; Hanslmayr et al., 2008; Liotti, Woldorff, Perez, \& Mayberg, 2000; West, 2003). In contrast, the SP probably represents both conflict detection and resolution and seems to be generated by a broader range of neuronal modules (Liotti et al., 2000; West, 2003).

Our aim was to investigate whether trait anxiety is associated with potential trial-to-trial adaptations in the N400 and SP range, indicating dynamic adjustments in cognitive control. Specifically, we assumed that if trait anxiety is positively correlated with a reactive recruitment of control resources, as postulated in the ACT (Eysenck et al., 2007) and DMC (Braver et al., 2007; Fales et al., 2008) accounts, this might particularly influence the N400 and SP amplitude on those trials directly preceded by an incongruent target-distractor pairing. More precisely, a reactive engagement of the underlying neurocognitive mechanisms following an incongruent trial may increase the general sensitivity or activity of these mechanisms in a presetting manner, resulting in higher amplitudes in these two ERP components on the subsequent trial.

\section{METHOD}

\section{Subjects}

Seventy-one students ( 52 female; mean age $=22.2$ years, $S D=$ 2.6), who were native German speakers and had normal or correctedto-normal vision, participated in this study. The mental and physical health of all the subjects was checked by a brief self-disclosure form. All the subjects gave their written informed consent before any testing and received either a monetary incentive $(€ 10)$ or course credits. The present study was in accordance with the ethical norms of the Ethics Committee of the German Psychological Society.

\section{Self-Report Inventory}

A German version of Spielberger's State-Trait Anxiety Inventory (STAI; Laux, Glanzmann, Schaffner, \& Spielberger, 1981; Spielberger, Gorsuch, \& Lushene, 1970) was used to measure anxiety. This questionnaire comprises 40 mood-describing statements that subjects can agree or disagree with on a 4-point scale. Twenty items assess anxiety as a current emotional state (state anxiety), whereas the other 20 items ask for the general frequency of such states (trait anxiety).

\section{Gender Stroop Task}

In this paradigm, single face-word stimuli were centrally presented on a computer screen (see Figure 1). The stimulus pool consisted of six female and six male grayscale faces with a neutral expression (Lundqvist \& Litton, 1998). The German words "Mann" (man) and "Frau" (woman) were written across the faces in red letters, resulting in a congruent or incongruent matching of the faceword pairing. The subjects were asked to discriminate the gender of the faces by pressing one of two response buttons (male $=$ left index finger, female $=$ right index finger) as quickly and accurately as possible. No specific instruction was given with respect to the word 


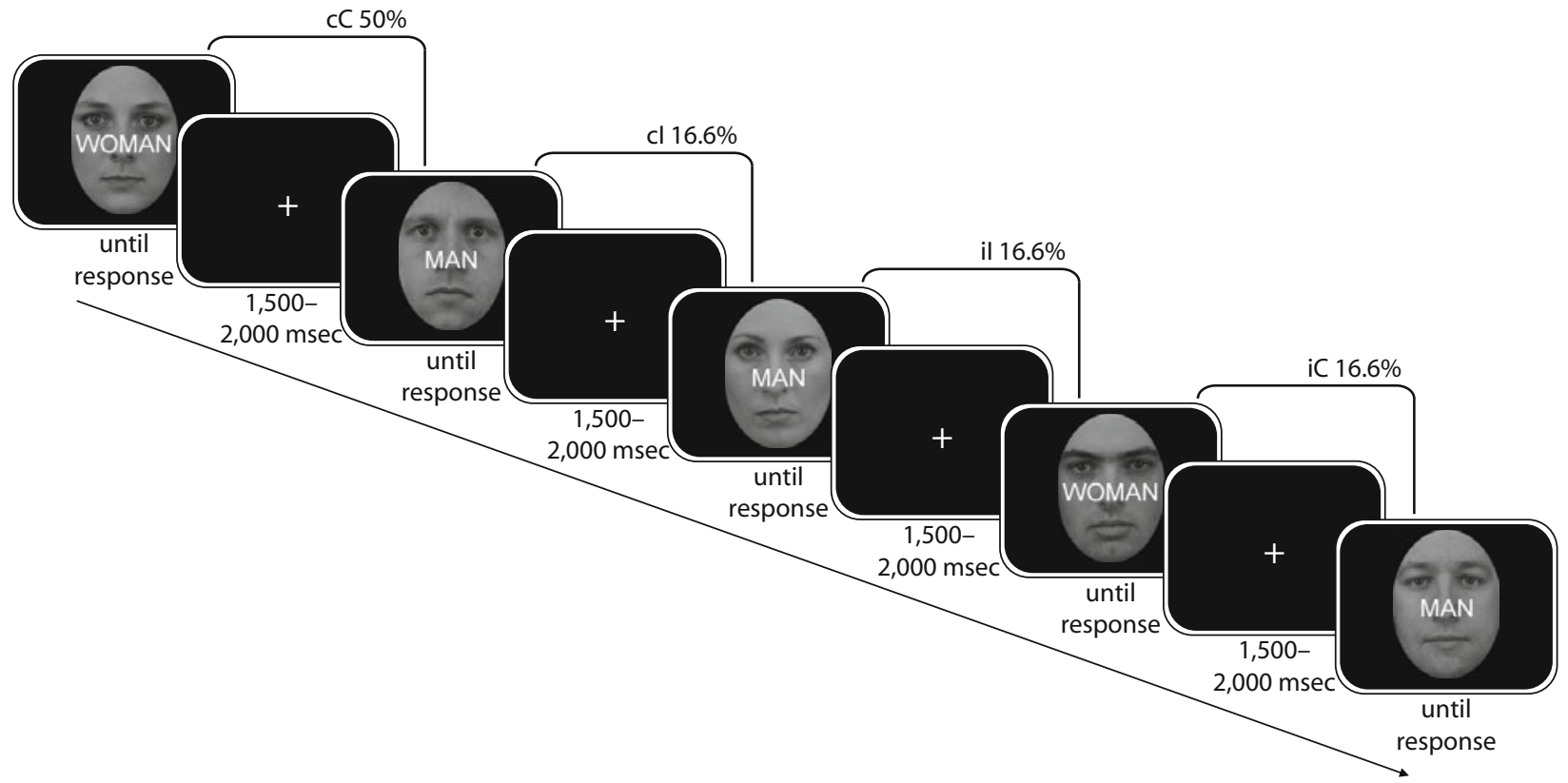

Figure 1. Schematic depiction and frequencies of trial sequences in the gender discrimination Stroop task. In the original task, distracting words were presented in red font. $\mathrm{cC}$, congruent trial preceded by congruent trial; cI, incongruent trial preceded by congruent trial; iI, incongruent trial preceded by incongruent trial; $\mathrm{iC}$, congruent trial preceded by incongruent trial.

stimuli. The complete paradigm comprised eight blocks of 72 trials each, resulting in a total of 576 trials. On two thirds (384) of the trials, the face-word pairing was congruent, whereas the other trials (192) were incongruent. Preceding the critical trials, 12 (Block 1) or 4 (Blocks 2-8) practice trials were presented, using two extra faces. The pseudorandomized trial order was the same for all the subjects and was characterized by the following restrictions: Each face was presented six times per block (four times congruent, two times incongruent), with no direct repetitions of the same face. Thirty-six trials of each block were congruent and preceded by other congruent trials ( $c C$ trials). The other trials were congruent preceded by incongruent trials ( $i C$ trials; 12 trials per block), incongruent preceded by congruent trials ( $c I$ trials; 12 trials per block), and incongruent preceded by incongruent trials (iI trials; 12 trials per block). Thus, the following total trial numbers resulted: $\mathrm{cC}=288, \mathrm{cI}=96, \mathrm{iC}=$ 96 , iI $=96$. The four trial type categories were matched with respect to the gender of the face stimuli (50\% male, $50 \%$ female) and the frequency of potential negative-priming trials (Neill, 1978; target value on trial $x$ is the same as distractor value on trial $x-1 ; 50 \%$ in each category). Moreover, the frequency of direct repetitions of facial gender was equal $(50 \%)$ for the congruent and incongruent conditions in each block.

On each trial, the stimulus was presented until a response was given. The interstimulus interval varied randomly between 1,500 and 2,000 msec. During this time, a central fixation cross was presented. Each block was followed by a short break of about $30 \mathrm{sec}$. The stimuli had a size of $4.5 \times 6.5 \mathrm{~cm}$. The subjects were seated in a comfortable chair at a distance of $80 \mathrm{~cm}$ from the screen. All the stimuli were presented on a 15 -in. monitor with a black background. Stimulus presentation and response recording were controlled by a Pentium- (Intel Corp., Santa Clara, CA) based PC and Presentation 12.2 software (Neurobehavioral Systems, Albany, CA). Responses were given on a customary response pad (Cedrus RB-730, Cedrus Corp., San Pedro, CA) with a 1-msec RT resolution. Statistical analyses were conducted using SPSS 15.0 (SPSS Inc., Chicago).

\section{Treatment and Analysis of Behavioral Data}

All error $(M=5.2 \%, S D=4.8)$ and posterror $(M=4.6 \%, S D=$ $3.8)$ trials were excluded from RT analysis. The same applied to cor- rect trials with RTs below $200 \mathrm{msec}(M<0.001 \%, S D<0.001)$ or above $2,000 \mathrm{msec}(M=0.3 \%, S D=0.7)$. Individual mean response latencies were then calculated for the four trial type categories $(\mathrm{cC}, \mathrm{cI}, \mathrm{iC}$, and iI). A three-way ANOVA $(2 \times 2 \times 3)$ was conducted with the within-subjects factors of previous trial (congruent, incongruent) and current trial (congruent, incongruent) and the betweensubjects factor of trait anxiety [low $(n=24)$, medium $(n=25)$, high $(n=22)$ ]. For the last factor, the complete sample was split into three groups, using the $33 \mathrm{rd}$ and 66 th percentiles of the STAI trait anxiety scores. Moreover, to control for potential feature repetition effects (Mayr, Awh, \& Laurey, 2003), the same ANOVA was conducted using only complete alternations in sequences of targetdistractor pairing for the iI and $\mathrm{cC}$ trials (e.g., previous trial, target $=$ male face, distractor = "Frau"; current trial: target = female face, distractor = "Mann").

For statistical analysis of performance accuracy, the hit rates (HRs) for the four trial type categories were first arcus-sinus transformed and then entered into a three-way ANOVA $(2 \times 2 \times 3)$ with the factors described above.

Furthermore, we calculated single-score indices of interference ( $\mathrm{RT}$ interference $=\mathrm{RT}$ incongruent $-\mathrm{RT}$ congruent; HR interference $=$ HR congruent - HR incongruent) and conflict adaptation [RT adaptation score $=[(\mathrm{iC}-\mathrm{cC})+(\mathrm{cI}-\mathrm{iI})] / 2$; HR adaptation score $=[(\mathrm{cC}-\mathrm{iC})+(\mathrm{iI}-\mathrm{cI})] / 2]$ on the basis of findings of previous studies (Egner, 2007).

\section{ERP Recordings and Analyses}

While the subjects completed the behavioral task, the EEG (analog band-pass, $0.1-250 \mathrm{~Hz}$; sampling rate, $1000 \mathrm{~Hz}$ ) was recorded from 29 scalp sites (Fp1, Fp2, F7, F3, Fz, F4, F8, FC1, FC2, T7, C3, Cz, C4, T8, TP9, CP1, CP2, TP10, P7, P3, Pz, P4, P8, PO9, O1, Oz, $\mathrm{O} 2$, PO10) using active $\mathrm{Ag} / \mathrm{AgCl}$ electrodes (actiCAP 32Ch; Brain Products, Gilching, Germany) and a BrainAmpDC amplifier (Brain Products). All impedances were kept below $5 \mathrm{k} \Omega$. During recording, all electrodes were referenced to the nose tip. The horizontal and vertical electrooculograms were recorded for detection of blinks and eye movements.

The data were processed offline, using Brain Vision Analyzer 2.0 software (Brain Products). First, the data were digitally rereferenced 
Table 1

Mean Values ( $\pm S D$ s) for Trait and State Anxiety, Gender Ratios, and Mean Age $( \pm S D)$ in the Complete Sample and in the Three Trait Anxiety Subgroups

\begin{tabular}{|c|c|c|c|c|c|c|c|}
\hline \multirow[b]{2}{*}{ Group } & \multicolumn{2}{|c|}{$\begin{array}{c}\text { Trait } \\
\text { Anxiety }{ }^{A, B, C} \\
\end{array}$} & \multicolumn{2}{|c|}{$\begin{array}{c}\text { State } \\
\text { Anxiety a,b,c } \\
\end{array}$} & \multirow{2}{*}{$\begin{array}{c}\text { Gender } \\
\text { Ratio } \\
\text { (Women/Men) }\end{array}$} & \multicolumn{2}{|c|}{ Age } \\
\hline & $M$ & $S D$ & $M$ & $S D$ & & $M$ & $S D$ \\
\hline Complete sample $(N=71)$ & 37.5 & 6.9 & 37.1 & 6.2 & $52 / 19$ & 22.2 & 2. \\
\hline Low trait anxiety $(n=24)$ & 30.7 & 3.1 & 34.0 & 3.9 & $17 / 7$ & 21.9 & 2. \\
\hline Medium trait anxiety $(n=25)$ & 40.0 & 1.2 & 37.3 & 1.2 & $18 / 7$ & 22.5 & 3.2 \\
\hline High trait anxiety $(n=22)$ & 45.7 & 4.9 & 40.3 & 6.7 & $17 / 5$ & 22.2 & 2.5 \\
\hline
\end{tabular}

Note-The superscripts A, B, and C indicate significant $(p<.05)$ differences between the groups: Alow versus medium, ${ }^{B}$ medium versus high, ${ }^{C}$ low versus high. In the same manner, the superscripts $\mathrm{a}$ and $\mathrm{b}$ indicate marginal differences $(p<.1)$.

to the averaged mastoid electrodes and were filtered using a $20-\mathrm{Hz}$ low-pass, a $0.15-\mathrm{Hz}$ high-pass, and a $50-\mathrm{Hz}$ notch filter. The EEG was then segmented (stimulus locked; -200 to $800 \mathrm{msec}$ ) for the four trial categories (cC, cI, iC, and iI), and epochs were semiautomatically scanned for artifacts and were discarded if applicable. For further analyses, all error and posterror trials were excluded, as well as all trials with RTs below $200 \mathrm{msec}$ and above 2,000 msec. These procedures resulted in the following mean $( \pm S D)$ trial numbers: $\mathrm{cC}$, 195.2 (42.6); cI, 62.3 (14.4); iC, 59.6 (13.7); iI, 60.7 (14.1). For each subject and each trial type, a minimum of 20 trials were available for averaging. Data segments were baseline corrected using the 200msec prestimulus epochs, and individual stimulus-locked average waves were calculated.

For statistical analyses, mean amplitudes were calculated for pooled electrode sites (frontal, F3, Fz, F4; central, C3, Cz, C4; parietal, P3, Pz, P4) and two time windows (350-450 msec [N400] and 500-800 msec [SP]). These epochs were chosen on the basis of previous ERP studies with the Stroop task and on visual inspection of the grand average waveforms. The mean amplitudes for each of the time windows were entered into a four-way ANOVA with the within-subjects factors of previous trial (congruent, incongruent), current trial (congruent, incongruent), and electrode site (frontal, central, parietal) and the between-subjects factor of trait anxiety (low, medium, high).

Moreover, we calculated single-score indices for the N400 and SP, reflecting the conflict effect (incongruent minus congruent; N400 conflict score and SP conflict score) and the context effect of the preceding trial $\{[(\mathrm{iC}-\mathrm{cC})+(\mathrm{iI}-\mathrm{cI})] / 2 ; \mathrm{N} 400$ context score and SP context score $\}$. The latter was based on the assumption that when the reactive recruitment of top-down control is exerted in a trial-totrial manner, this should manifest in an additional, presetting influence on the N400 or SP as a function of the preceding congruency and independently of current congruency. The conflict and context scores were correlated with trait anxiety and behavioral indices of interference and conflict adaptation.

\section{RESULTS}

\section{Sample Characteristics}

In Table 1, sample characteristics (age, gender distribution, anxiety scores) are listed. There were no differences between the three trait anxiety groups with respect to gender ratio $\left[\chi^{2}(2)=0.27, p=.87\right]$ and age $[F(2,68)=0.28$, $p=.75]$. Moreover, in our sample, women did not show higher trait anxiety scores than did men $[t(69)=0.40$, $p=.69]$. There was a moderate relation between trait and state anxiety $(r=.40)$, resulting in a substantial effect of the dispositional group factor on state anxiety $[F(2,68)=$ $6.94, p<.01]$. Post hoc tests revealed that there were strong state anxiety differences between the high- and low-trait-anxiety groups $(p<.001)$ and marginal differences between the medium- and low- $(p=.052)$ and the medium- and high- $(p=.073)$ trait-anxiety groups. In the subsequent analyses, we therefore controlled for state anxiety whenever a substantial effect of trait anxiety was detected.

\section{Behavioral Interference and Conflict Adaptation}

For all the ANOVAs mentioned below, GreenhouseGeisser-corrected statistics were used in cases of violated sphericity assumption. Behavioral results are depicted in Figure 2. Analysis of response latencies revealed that the subjects reacted more quickly in the congruent condition (mean RT, $582 \mathrm{msec}$ ) than in the incongruent condition [mean RT, $651 \mathrm{msec}$; main effect of current trial, $F(1,68)=$ $309.02, p<.001]$. This main effect was qualified by a significant interaction of current trial and previous trial congruency $[F(1,68)=27.36, p<.001]$, arguing for a substantial conflict adaptation effect [pairwise comparisons: $\mathrm{cC}<\mathrm{iC}<\mathrm{iI}<\mathrm{cI}($ all $p$ s $<.05$ ); see Figure 2A]. Thus, across all subjects, the previous trial context had a crucial effect on the response speed on the current trial. More precisely, the congruency effect was diminished in the context of a preceding incongruent trial. Importantly, this pattern was stable when we controlled for potential feature repetition effects [see Figure 2B; previous trial $\times$ current trial, $F(1,68)=34.74, p<.001$; pairwise comparisons, $\mathrm{cC}<$ $\mathrm{iC}<\mathrm{iI}<\mathrm{cI}($ all $p \mathrm{~s}<.05)$ ]. We found neither a significant main effect of trait anxiety nor a substantial interaction with the other factors (all $p \mathrm{~s}>.36$; see Table 2).

Performance was also significantly more accurate in the congruent condition (mean HR, 97.3\%), as compared with the incongruent condition (mean HR, 92.2\%) [main effect of current trial: $F(1,68)=109.28, p<.001]$. Moreover, the subjects generally made fewer errors in the context of a preceding incongruent trial (mean HR, 95.3\%), as compared with a preceding congruent trial (mean HR, 94.0\%) [main effect of previous trial, $F(1,68)=13.83, p<.001$ ] These two main effects were qualified by a significant current trial $\times$ previous trial interaction $[F(1,68)=24.61$, $p<.001$; pairwise comparisons, $\mathrm{cC}>\mathrm{iC}>\mathrm{iI}>\mathrm{cI}$ (all $p \mathrm{~s}<.05$ ); see Figure $2 \mathrm{C}$ ], indicating the same pattern of conflict adaptation as that reported for response latencies. Again, no significant effect of trait anxiety was found (all $p$ s > .36; see Table 2). 

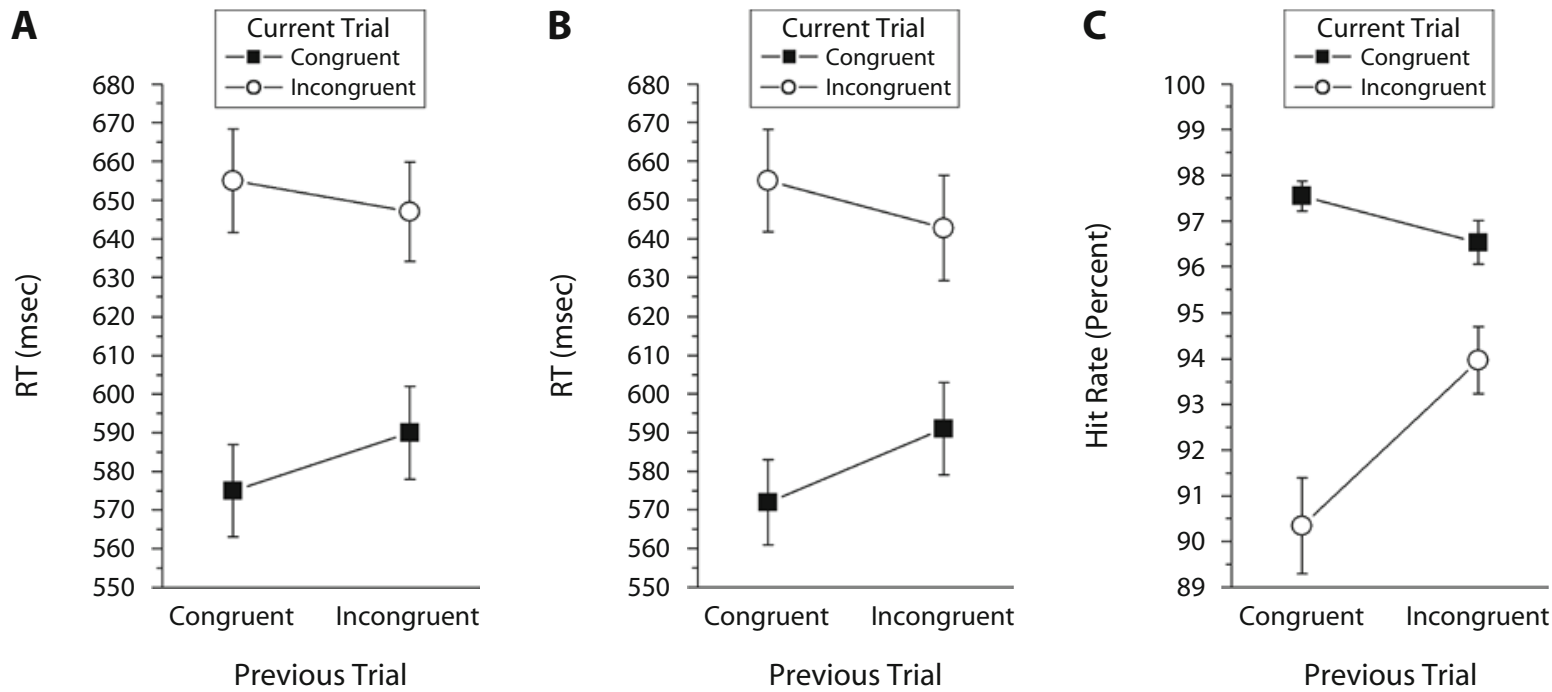

Figure 2. Behavioral data plotted for the interaction of current trial and previous trial congruency. (A) Mean reaction times (RTs; $\pm S E$ s), including all trials. (B) Mean RTs ( $\pm S E$ s) corrected for feature repetition effects by including only complete target-distractor alternations on $\mathrm{cC}$ and iI trials. (C) Mean hit rates ( $\pm S E$ s) in percentages. However, statistical analyses were conducted using arcus-sinus transformed hit rates.

Table 2

Mean Reaction Times $( \pm S D$ s; in Milliseconds) and Hit Rates (in Percentages) for the Three Trait Anxiety Groups

\begin{tabular}{|c|c|c|c|c|c|c|c|c|}
\hline \multirow[b]{3}{*}{ Group } & \multicolumn{8}{|c|}{ Trial Type } \\
\hline & \multicolumn{2}{|c|}{$\mathrm{cC}$} & \multicolumn{2}{|c|}{$\mathrm{iC}$} & \multicolumn{2}{|c|}{ iI } & \multicolumn{2}{|c|}{$\mathrm{cI}$} \\
\hline & $M$ & $S D$ & $M$ & $S D$ & $M$ & $S D$ & $M$ & $S D$ \\
\hline \multicolumn{9}{|l|}{ Low Trait Anxiety } \\
\hline Reaction time & 592 & 134 & 604 & 135 & 662 & 129 & 670 & 145 \\
\hline Hit rate & 97.9 & 1.6 & 97.0 & 2.7 & 94.6 & 4.6 & 90.5 & 6.5 \\
\hline \multicolumn{9}{|c|}{ Medium Trait Anxiety } \\
\hline Reaction time & 582 & 78 & 601 & 85 & 661 & 104 & 660 & 93 \\
\hline Hit rate & 97.7 & 2.1 & 96.8 & 2.7 & 93.6 & 7.9 & 91.4 & 11.1 \\
\hline \multicolumn{9}{|c|}{ High Trait Anxiety } \\
\hline Reaction time & 551 & 72 & 567 & 70 & 618 & 86 & 633 & 91 \\
\hline Hit rate & 97.0 & 4.1 & 95.8 & 6.0 & 93.7 & 5.5 & 89.2 & 7.9 \\
\hline
\end{tabular}

Note- $\mathrm{cC}$, congruent trials preceded by congruent trials; iC, congruent preceded by incongruent; iI, incongruent preceded by incongruent; cI, incongruent preceded by congruent.

\section{N400}

In the 350- to 450-msec time window, we detected the N400 as a substantial negative-going deflection in the incongruent condition, relative to the congruent condition $[F(1,68)=68.00, p<.001]$, which was especially pronounced at parietal electrodes $[F(1.9,81.3)=6.39, p<$ $.05]$. Mean amplitudes were also more negative in the context of an incongruent preceding trial [main effect of previous trial: $F(1,68)=13.50, p<.001]$-again, especially at parietal sites $[F(1.2,84.0)=11.76, p<.001]$. Moreover, we detected a significant electrode site $\times$ current trial $\times$ previous trial interaction $[F(1.2,81.8)=8.08, p<$ $.01]$ : The effect of previous trial congruency was higher for currently congruent, as compared with incongruent, trials, and this interaction was especially pronounced at parietal sites.

Importantly, the effect of previous trial congruency was moderated by trait anxiety [previous trial $\times$ trait anxiety:
$F(2,68)=3.60, p<.05]$. As can be seen in Figure 3A, the previous trial congruency effect was most pronounced in the group of high-trait-anxious subjects. Accordingly, pairwise comparisons indicated that only in the group of high-trait-anxious subjects could a significant effect of previous trial be detected (high trait anxiety, $p<.001$; medium trait anxiety, $p=.38$; low trait anxiety, $p=$ .24). This interaction between trait anxiety and previous trial congruency tended to be particularly pronounced at the parietal site $[F(2.5,84.0)=2.54, p=.07]$ and remained stable when we entered state anxiety as a covariate $[F(2,67)=3.64, p<.05]$.

\section{SP}

The most obvious effect in the 500- to 800 -msec time window was a sustained positive deflection on incongruent trials, relative to congruent trials $[F(1,68)=43.63$, $p<.001]$, which was especially pronounced at parietal 
A

Complete Sample $(\boldsymbol{N}=71)$

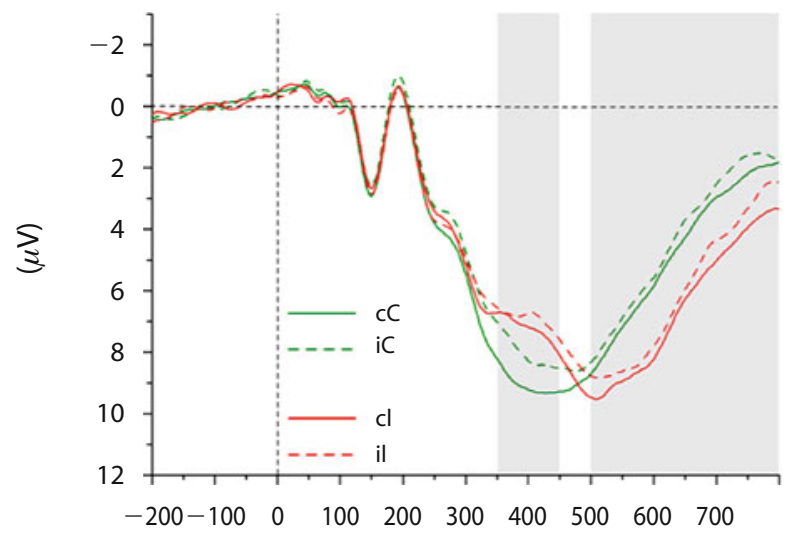

(msec)

Low Trait Anxiety $(n=24)$

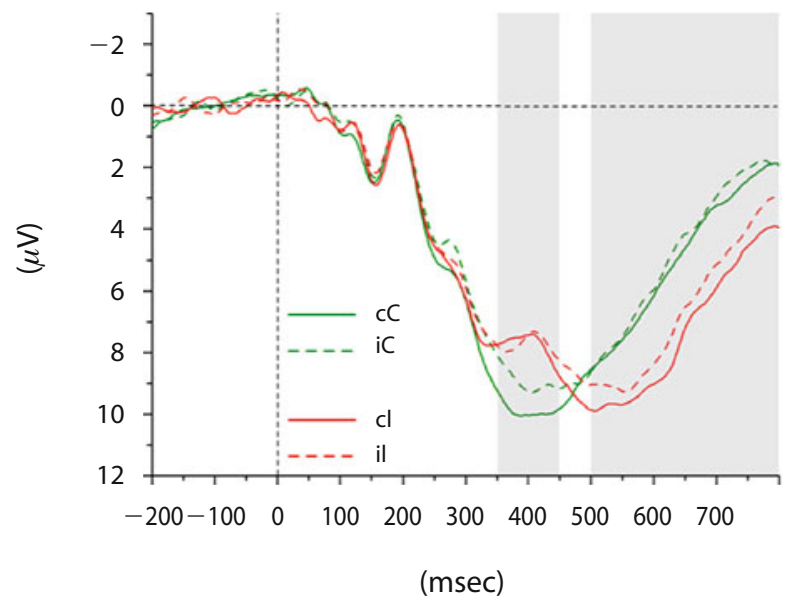

B

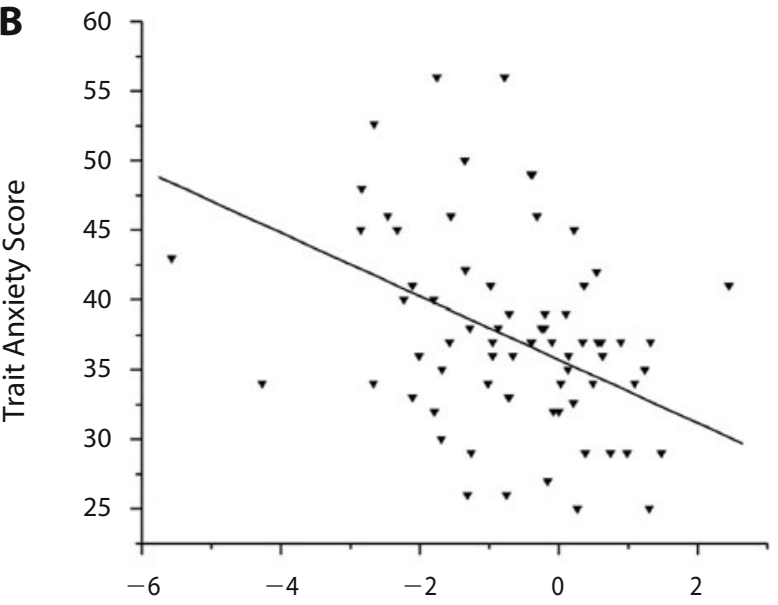

N400 Context Score $(\mu \mathrm{V})$

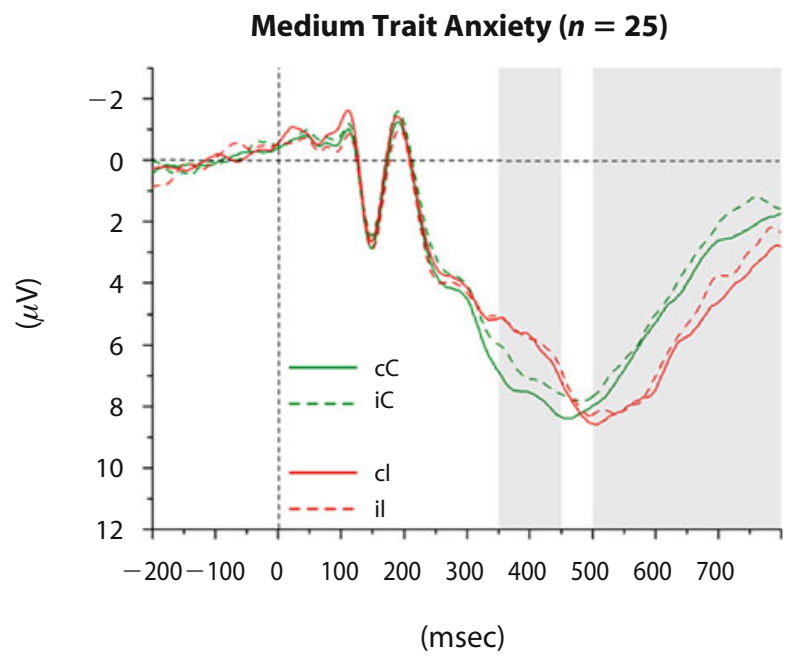

High Trait Anxiety ( $n=22$ )

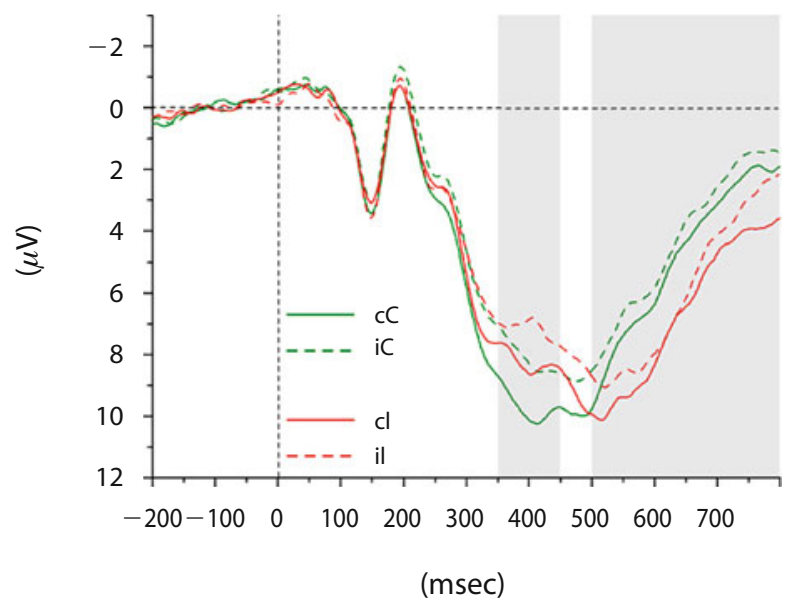

$[(\mathrm{iC}-\mathrm{cC})+(\mathrm{il}-\mathrm{cl})] / 2$ $350-450 \mathrm{msec}$

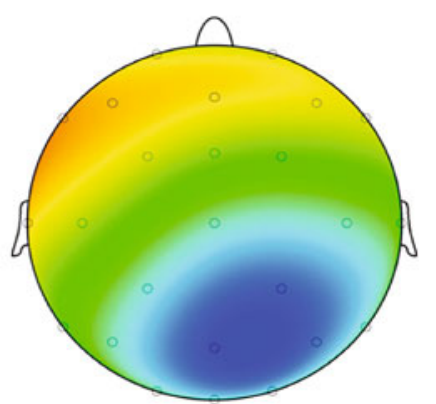

$0.0 \mu \mathrm{V}$

Figure 3. (A) ERP at the parietal site (pool of P3, Pz, and P4) plotted depending on the interaction of current trial (green $=$ congruent, red = incongruent) and previous trial (solid lines = congruent, dashed lines $=$ incongruent) congruency for the complete sample and for high-, medium-, and low-anxious subjects separately. Negativity is plotted upward. (B) Topographical map for the voltage distribution of the $\mathbf{N 4 0 0}$ context score in the complete sample and a scatterplot for the relation between this score and trait anxiety. For further explanation of abbreviations, see the text, p. 374 . 
electrodes $[F(1.2,84.9)=114.94, p<.001$; see Figure $3 \mathrm{~A}]$. The mean amplitude in this epoch was also influenced by previous trial congruency $[F(1,68)=5.99, p<$ $.05]$ and by the previous trial $\times$ electrode site interaction $[F(1.2,78.5)=6.64, p<.01]$ : Incongruency of the previous trial was associated with a small but significant negative deflection on the current trial, especially pronounced at the parietal site. Moreover, we found a significant trait anxiety $\times$ current trial $\times$ electrode site interaction $[F(2.5,84.9)=5.34, p<.01]$. However, inspection of this complex interaction yielded no linear effects of trait anxiety on the conflict-related SP but a small difference between the three groups at the frontal site (i.e., a slightly higher difference between the congruent and incongruent conditions in the medium-trait-anxiety group, as compared with the high- and low-trait-anxiety groups), which is rather hard to interpret.

\section{Correlations Among Trait Anxiety, Behavioral, and Electrophysiological Indices}

Replicating the above-mentioned results, we detected a significant correlation between trait anxiety and the context effect in the N400 range at the parietal $(r=-.32$, $p<.01$; see Figure 3B) and central $(r=-.29, p<.05)$ sites. Thus, an increase in anxiety was associated with an increase in the effect of previous trial congruency on the N400 amplitude. This pattern remained stable when the effects of state anxiety and behavioral conflict adaptation were partialized out (parietal, $r=-.27, p<.05$; central, $r=-.28, p<.01)$. Trait anxiety correlated neither with the N400 conflict score nor with any index of the SP.

When analyzing the relations between the psychophysiological conflict scores and the behavioral interference scores, we detected one substantial outlier by means of standard methods (residual plots, Cook's distance). ${ }^{1}$ This subject was therefore excluded from the respective analyses. The N400 conflict score at the frontal and central sites was associated with interference in accuracy (frontal, $r=$ $-.28, p<.05$; central, $r=-.28, p<.05$ ), but not in the RT domain. Thus, an increase in the conflict-related N400 was associated with an increase in accuracy interference. Moreover, the N400 context score at the parietal site was correlated with conflict adaptation in the HRs $(r=-.28, p<.05)$. The SP conflict score was not related to behavioral interference. However, the SP context score at the parietal site was correlated with behavioral conflict adaptation in the HRs $(r=-.29, p<.05)$. Therefore, the context effect of the previous trial in the N400 and SP time windows was related to conflict adaptation in accuracy. That is, the stronger the negative-going deflections in the N400 and SP range in the context of an incongruent preceding trial, the stronger the dynamic adjustments in the HRs.

\section{DISCUSSION}

To our knowledge, this study is the first to investigate potential links between anxiety as a dispositional trait and dynamic adjustments in neuronal correlates of cognitive control. In sum, the results were as follows. First, the behavioral data confirmed that strong stimulus-response conflicts were evoked in our gender discrimination Stroop task. The interference in accuracy and response latencies was influenced by the sequential order of targetdistractor congruency, indicating trial-to-trial variations in control. Second, in our ERP analyses, we were able to replicate the results of previous studies by identifying two wave components crucially linked to stimulus-response conflicts-namely, the N400 and the SP. The observed correlations between these components and behavioral indices of interference and conflict adaptation argue for the functional relevance of the underlying neurocognitive mechanisms in conflict processing. The N400, in particular, seems to reflect functioning of a conflict-monitoring system (an increase in behavioral interference was associated with an increase in the N400 conflict score), which is presumably located in the AACC (Badzakova-Trajkov et al., 2009; Hanslmayr et al., 2008; Liotti et al., 2000; West, 2003; West, Jakubek, Wymbs, Perry, \& Moore, 2005). Third, and probably most important, our results indicate that trait anxiety is linked to the way this system dynamically adjusts to changes in cognitive demands. Independently of current trial congruency, highly anxious subjects showed an additional negative-going deflection in the N400 time window in the context of a preceding incongruent trial, probably indicating a reactive engagement of the conflict monitor as a direct response to an acute need for top-down guidance. This assumption is in accordance with recent theoretical frameworks (Braver et al., 2007, p. 95) and is also supported by other studies, pointing to a crucial link between dispositional anxiety and functioning of the dACC. For example, Eisenberger, Lieberman, and Satpute (2005) reported a positive correlation between neuroticism (an anxiety-related personality trait) and dACC reactivity in an oddball task. In detail, subjects scoring high on neuroticism showed an increased dACC response to infrequent stimuli (oddballs) that deviated from the expected predominant stimulus. In another study, high trait anxiety was found to be linked to increased dACC reactivity to ambiguous affective stimuli (Simmons et al., 2008). Moreover, clinical studies have pointed to an increased dACC activity in patients with obsessive-compulsive disorder during response conflicts (Maltby, Tolin, Worhunsky, O'Keefe, \& Kiehl, 2005; Ursu, Stenger, Shear, Jones, \& Carter, 2003) and in generalized anxiety disorder patients during states of worry (Paulesu et al., 2010). In addition to these fMRI findings, several ERP studies have reported an increased error-related negativity (ERN) in highly anxious subjects (Boksem, Tops, Wester, Meijman, \& Lorist, 2006; Hajcak, McDonald, \& Simons, 2003; Ladouceur, Dahl, Birmaher, Axelson, \& Ryan, 2006; Olvet \& Hajcak, 2009). The ERN also reflects processes of performance monitoring and is probably generated by the dACC (Dehaene, Posner, \& Tucker, 1994; Gehring, Goss, Coles, Meyer, \& Donchin, 1993). Using a go/no-go task, two other recent ERP studies also showed positive correlations between self-reported behavioral inhibition (an anxiety-related 
trait) and electrophysiological indicators of conflict processing in the dACC (Amodio, Master, Yee, \& Taylor, 2008; Wacker, Chavanon, Leue, \& Stemmler, 2010). This entire research indicates that dispositional anxiety is crucially linked to a neurocognitive system that is primarily engaged in discrepancy monitoring and detection at various levels of information processing. Furthermore, our findings demonstrate that trait anxiety is also associated with the way this system transiently reacts and adjusts to recent demands. Thereby, the present study not only confirms the reactive control mode in highly anxious subjects postulated by others (Braver et al., 2007; Fales et al., 2008), but further specifies this mode by elucidating its dynamic nature.

These findings may, therefore, have some important implications for future research on both anxiety and cognition. First of all, further studies on potential links between trait anxiety and executive functions should also consider sequential variations in control, since substantial variance in neuronal activity is probably disregarded by ignoring such dynamics. Accordingly, the abovementioned link between anxiety and the ERN may also vary as a function of dynamic adjustments in cognitive control. Future approaches addressing this topic should apply other behavioral paradigms evoking higher error rates. Moreover, future studies may also take macroadjustments in control into account. For example, the reported link between trait anxiety and the context effect on the N400 may also vary across time, depending on the general engagement with the task (Luu, Collins, \& Tucker, 2000). In a post hoc analysis of our data, we tested for this possibility by comparing the first and the second halves of our paradigm. Although we found no direct evidence for such effects, future studies with higher trial numbers may further investigate this issue, allowing for a better temporal resolution of macrovariations in cognitive processing. Generally, future research on potential links between trait anxiety and the processing of emotional material may also consider dynamic adjustments in cognition. For instance, the frequently documented attentional bias for threatening stimuli in highly anxious subjects may be modulated by the specific sequential alternation of threatening and neutral stimuli (Bar-Haim, Lamy, Pergamin, BakermansKranenburg, \& van IJzendoorn, 2007; Bishop, 2007; Weierich, Treat, \& Hollingworth, 2008).

Our findings may also be interpreted in the context of motivational approaches to personality. In their revised reinforcement sensitivity theory, J. A. Gray and McNaughton (2000) postulated that individual differences in trait anxiety are caused by differences in sensitivity to motivational conflicts. For example, being forced to choose between two undesirable options will lead to an avoidance-avoidance conflict, and there is evidence that the $\mathrm{dACC}$ is also crucially involved in the processing of such motivational conflicts (Blair et al., 2006). Given that our results are transferable to the motivational domain (see also Boksem, Tops, Kostermans, \& De Cremer, 2008; Wacker et al., 2010), one could assume that trait anxiety is not related just to general sensitivity, but also to the dynamic adjustment of the motivational conflict detector.

It should be noted that our argumentation, thus far, is based solely on the assumption that the context effect in the N400 range reflects a reactive recruitment of a conflict-monitoring mechanism. However, the additional negative-going deflection in the context of a preceding incongruent trial could also mirror other cognitive mechanisms, such as implementation of top-down control by the DLPFC (e.g., Kerns et al., 2004) and/or frontoparietal attention networks (Posner \& Dehaene, 1994). This could also explain the correlation between the N400 context score and the conflict adaptation effect in accuracy, in that, for example, a stronger implementation of attentional control leads to stronger conflict adaptation in behavior. However, the relation between electrophysiological and behavioral indices is not necessarily causal in nature but might also point to a shared underlying factor. Both the potential reactive recruitment of a conflict-monitoring mechanism and the conflict adaptation in accuracy might be the consequence of a common third factor (e.g., implementation of control by the DLPFC). Certainly, more research is needed to disentangle the complex interrelation between neurocognitive and behavioral mechanisms of adjustments in control.

In sum, the present study goes beyond previous approaches to trait anxiety and executive functions (e.g., Bishop, 2009; Fales et al., 2008; J. R. Gray \& Braver, 2002; J. R. Gray et al., 2005) by considering trial-to-trial adjustments in cognitive control. We successfully demonstrated that trait anxiety is associated with context-dependent variations in the ERP, which presumably reflect the reactive recruitment of a neuronal conflict-monitoring mechanism. Thus, individual differences in dispositional anxiety appear to be linked to the way the cognitive system reacts and adapts to an acute need for top-down guidance. More studies are needed to elucidate how the reported cognitive pattern contributes to the development and symptomatic characteristics (e.g., cognitive, affective, and behavioral) of anxiety. Generally, our findings endorse the consideration of personality constructs in research on top-down control, and, therefore, future research in this domain may also take such systematic variations between human beings into account to further our understanding of complex cognition.

\section{AUTHOR NOTE}

Correspondence concerning this article should be addressed to R. Osinsky, Department of Psychology, Justus Liebig University Giessen, Otto-Behaghel-Str. 10 F, 35394 Giessen, Germany (e-mail: roman .osinsky@psychol.uni-giessen.de).

\section{REFERENCES}

Amodio, D. M., Master, S. L., Yee, C. M., \& Taylor, S. E. (2008). Neurocognitive components of the behavioral inhibition and activation systems: Implications for theories of self-regulation. Psychophysiology, 45, 11-19.

Ansari, T. L., Derakshan, N., \& Richards, A. (2008). Effects of anxiety on task switching: Evidence from the mixed antisaccade task. $C o g$ nitive, Affective, \& Behavioral Neuroscience, 8, 229-238. 
Badzakova-Trajkov, G., Barnett, K. J., Waldie, K. E., \& Kirk, I. J. (2009). An ERP investigation of the Stroop task: The role of the cingulate in attentional allocation and conflict resolution. Brain Research, 1253, 139-148

Bar-Haim, Y., Lamy, D., Pergamin, L., Bakermans-Kranenburg, M. J., \& VAN IJZENDOORN, M. H. (2007). Threat-related attentional bias in anxious and nonanxious individuals: A meta-analytic study. Psychological Bulletin, 133, 1-24.

BisHop, S. J. (2007). Neurocognitive mechanisms of anxiety: An integrative account. Trends in Cognitive Sciences, 11, 307-316.

Bishop, S. J. (2009). Trait anxiety and impoverished prefrontal control of attention. Nature Neuroscience, 12, 92-98.

Blair, K., Marsh, A. A., Morton, J., Vythilingam, M., Jones, M., Mondillo, K., ET AL. (2006). Choosing the lesser of two evils, the better of two goods: Specifying the roles of ventromedial prefrontal cortex and dorsal anterior cingulate in object choice. Journal of Neuroscience, 26, 11379-11386.

Boksem, M. A. S., Tops, M., Kostermans, E., \& De Cremer, D. (2008). Sensitivity to punishment and reward omission: Evidence from errorrelated ERP components. Biological Psychology, 79, 185-192.

Boksem, M. A. S., Tops, M., Wester, A. E., Meijman, T. F., \& Lorist, M. M. (2006). Error-related ERP components and individual differences in punishment and reward sensitivity. Brain Research, 1101, 92-101.

Botvinick, M. M., Braver, T. S., Barch, D. M., Carter, C. S., \& Cohen, J. D. (2001). Conflict monitoring and cognitive control. Psychological Review, 108, 624-652.

Braver, T. S., Barch, D. M., Gray, J. R., Molfese, D. L., \& Snyder, A. (2001). Anterior cingulate cortex and response conflict: Effects of frequency, inhibition and errors. Cerebral Cortex, 11, 825-836.

Braver, T. S., Gray, J. A., \& Burgess, G. C. (2007). Explaining the many varieties of working memory variation: Dual mechanisms of cognitive control. In A. R. A. Conway, C. Jarrold, M. J. Kane, A. Miyake, \& J. N. Towse (Eds.), Variation in working memory (pp. 76-106). Oxford: Oxford University Press.

Carter, C. S., Macdonald, A. M., Botvinick, M., Ross, L. L., Stenger, V. A., Noll, D., \& Cohen, J. D. (2000). Parsing executive processes: Strategic vs. evaluative functions of the anterior cingulate cortex. PNAS, 97, 1944-1948.

Carter, C. S., \& VAN VeEN, V. (2007). Anterior cingulate cortex and conflict detection: An update of theory and data. Cognitive, Affective, \& Behavioral Neuroscience, 7, 367-379.

Corbetta, M., \& Shulman, G. L. (2002). Control of goal-directed and stimulus-driven attention in the brain. Nature Reviews Neuroscience, 3, 201-215.

Dehaene, S., Posner, M. I., \& Tucker, D. M. (1994). Localization of a neural system for error-detection and compensation. Psychological Science, 5, 303-305.

Derakshan, N., \& Eysenck, M. W. (1998). Working memory capacity in high trait-anxious and repressor groups. Cognition \& Emotion, 12, 697-713.

EGNer, T. (2007). Congruency sequence effects and cognitive control. Cognitive, Affective, \& Behavioral Neuroscience, 7, 380-390.

Egner, T., \& Hirsch, J. (2005). Cognitive control mechanisms resolve conflict through cortical amplification of task-relevant information. Nature Neuroscience, 8, 1784-1790.

Eisenberger, N. I., Lieberman, M. D., \& Satpute, A. B. (2005). Personality from a controlled processing perspective: An fMRI study of neuroticism, extraversion, and self-consciousness. Cognitive, Affective, \& Behavioral Neuroscience, 5, 169-181.

EysencK, M. W., \& Byrne, A. (1992). Anxiety and susceptibility to distraction. Personality \& Individual Differences, 13, 793-798.

Eysenck, M. W., Derakshan, N., Santos, R., \& Calvo, M. G. (2007). Anxiety and cognitive performance: Attentional control theory. Emotion, 7, 336-353.

Fales, C. L., Barch, D. M., Burgess, G. C., Schaefer, A., Mennin, D. S., Gray, J. R., \& Braver, T. S. (2008). Anxiety and cognitive efficiency: Differential modulation of transient and sustained neural activity during a working memory task. Cognitive, Affective, \& Behavioral Neuroscience, 8, 239-253.

Gehring, W. J., Goss, B., Coles, M. G. H., Meyer, D. E., \& Don-
CHIN, E. (1993). A neural system for error detection and compensation. Psychological Science, 4, 385-390.

Gratton, G., Coles, M. G. H., \& Donchin, E. (1992). Optimizing the use of information: Strategic control of activation of responses. Journal of Experimental Psychology: General, 121, 480-506.

Gray, J. A., \& McNaughton, N. (2000). The neuropsychology of anxiety: An enquiry into the functions of the septo-hippocampal system (2nd ed.). New York: Oxford University Press.

Gray, J. R., \& Braver, T. S. (2002). Personality predicts workingmemory-related activation in the caudal anterior cingulate cortex. Cognitive, Affective, \& Behavioral Neuroscience, 2, 64-75.

Gray, J. R., Burgess, G. C., Schaefer, A., Yarkoni, T., Larsen, R. J., \& Braver, T. S. (2005). Affective personality differences in neural processing efficiency confirmed using fMRI. Cognitive, Affective, \& Behavioral Neuroscience, 5, 182-190.

HaJCAK, G., McDonald, N., \& Simons, R. F. (2003). Anxiety and errorrelated brain activity. Biological Psychology, 64, 77-90.

Hanslmayr, S., Pastötter, B., BäUml, K.-H., Gruber, S., WimBER, M., \& KLIMESCH, W. (2008). The electrophysiological dynamics of interference during the Stroop task. Journal of Cognitive Neuroscience, 20, 215-225.

Kerns, J. G., Cohen, J. D., MacDonald, A. W., III, Cho, R. Y., Stenger, V. A., \& CARTer, C. S. (2004). Anterior cingulate conflict monitoring and adjustments in control. Science, 303, 1023-1026.

Ladouceur, C. D., Dahl, R. E., Birmaher, B., Axelson, D. A., \& RYAN, N. D. (2006). Increased error-related negativity (ERN) in childhood anxiety disorders: ERP and source localization. Journal of Child Psycholology \& Psychiatry, 47, 1073-1082.

Larson, M. J., Kaufman, D. A. S., \& Perlstein, W. M. (2009). Neural time course of conflict adaptation effects on the Stroop task. Neuropsychologia, 47, 663-670.

Laux, L., Glanzmann, P., Schaffner, P., \& Spielberger, C. D. (1981). Das State-Trait-Angstinventar (STAI) [The State-Trait Anxiety Inventory]. Weinheim, Germany: Beltz.

Liotti, M., Woldorff, M. G., Perez, R., \& Mayberg, H. S. (2000). An ERP study of the temporal course of the Stroop interference effect. Neuropsychologia, 38, 701-711.

LundQVist, D., \& LitTon, J. E. (1998). The averaged Karolinska directed emotional faces-AKDEF (CD-ROM). Stockholm: Karolinska Institute, Department of Clinical Neuroscience.

Luu, P., Collins, P., \& Tucker, D. M. (2000). Mood, personality, and self-monitoring: Negative affect and emotionality in relation to frontal lobe mechanisms of error monitoring. Journal of Experimental Psychology: General, 129, 43-60.

MacDonald, A. W., Cohen, J. D., Stenger, V. A., \& Carter, C. S. (2000). Dissociating the role of the dorsolateral prefrontal and anterior cingulate cortex in cognitive control. Science, 288, 1835-1838.

MacLeod, C. M. (1991). Half a century of research on the Stroop effect: An integrative review. Psychological Bulletin, 109, 163-203.

Maltby, N., Tolin, D. F., Worhunsky, P., O’Keefe, T. M., \& Kiehl, K. A. (2005). Dysfunctional action monitoring hyperactivates frontalstriatal circuits in obsessive-compulsive disorder: An event-related fMRI study. NeuroImage, 24, 495-503.

MaYr, U., Awh, E., \& LaUREy, P. (2003). Conflict adaptation effects in the absence of executive control. Nature Neuroscience, 6, 450-452.

Miyake, A., Friedman, N. P., Emerson, M. J., Witzki, A. H., HowERTER, A., \& WAGER, T. D. (2000). The unity and diversity of executive functions and their contributions to complex "frontal lobe" tasks: A latent variable analysis. Cognitive Psychology, 41, 49-100.

NeILl, W. T. (1978). Decision processes in selective attention: Response priming in the Stroop color-word task. Perception \& Psychophysics, 23, 80-84

Olvet, D. M., \& HAJCAK, G. (2009). The effect of trial-to-trial feedback on the error-related negativity and its relationship with anxiety. Cognitive, Affective, \& Behavioral Neuroscience, 9, 427-433.

Paulesu, E., Sambugaro, E., Torti, T., Danelli, L., Ferri, F., ScIalfa, G., ET AL. (2010). Neural correlates of worry in generalized anxiety disorder and in normal controls: A functional MRI study. Psychological Medicine, 40, 117-124.

Posner, M. I., \& Dehaene, S. (1994). Attentional networks. Trends in Neurosciences, 17, 75-79. 
Scerif, G., Worden, M. S., Davidson, M., Seiger, L., \& Casey, B. J. (2006). Context modulates early stimulus processing when resolving stimulus-response conflict. Journal of Cognitive Neuroscience, 18, 781-792.

Simmons, A., Matthews, S. C., Feinstein, J. S., Hitchcock, C., PaULUS, M. P., \& STEIN, M. B. (2008). Anxiety vulnerability is associated with altered anterior cingulate response to an affective appraisal task. NeuroReport, 19, 1033-1037.

SPIElberger, C. D., Gorsuch, R. L., \& Lushene, R. E. (1970). STAI manual for the State-Trait Anxiety Inventory. Palo Alto, CA: Consulting Psychologists Press.

Stroop, J. R. (1935). Studies of interference in serial-verbal reaction. Journal of Experimental Psychology, 18, 643-662.

Ursu, S., Stenger, V. A., Shear, M. K., Jones, M. R., \& Carter, C. S. (2003). Overactive action monitoring in obsessive-compulsive disorder: Evidence from functional magnetic resonance imaging. Psychological Science, 14, 347-353.

VAN Veen, V., \& CARTER, C. S. (2002). The anterior cingulate as a conflict monitor: fMRI and ERP studies. Physiology \& Behavior, 77, 477-482.

Wacker, J., Chavanon, M.-L., Leue, A., \& Stemmler, G. (2010).
Trait BIS predicts alpha asymmetry and P300 in a go/no-go task. European Journal of Personality, 24, 85-105.

Weierich, M. R., Treat, T. A., \& Hollingworth, A. (2008). Theories and measurement of visual attentional processing in anxiety. Cognition \& Emotion, 22, 985-1018.

WEST, R. (2003). Neural correlates of cognitive control and conflict detection in the Stroop and digit-location tasks. Neuropsychologia, 41, $1122-1135$.

West, R., JakubeK, K., Wymbs, N., Perry, M., \& Moore, K. (2005). Neural correlates of conflict processing. Experimental Brain Research, 167, 38-48.

Wood, J., Mathews, A., \& Dalgleish, T. (2001). Anxiety and cognitive inhibition. Emotion, 1, 166-181.

\section{NOTE}

1. Scatterplots and statistics can be provided upon request.

(Manuscript received January 25, 2010; revision accepted for publication May 16, 2010.) 